\title{
Prevalence of biologic, behavioral and psychosocial determinant of tuberculosis in tuberculosis patients of Semnan city; a five-year cross-sectional study
}

\author{
Naim Sadat Kia' ${ }^{\circledR}$, Mohammad Nassaji Zavareh $^{2}{ }^{\circledR}$, Elnaz Sarkheil ${ }^{\mathbb{D}}$, Elaheh Ghods $^{1 *(\mathbb{D}}$ \\ ${ }^{1}$ Social Determinants of Health Research Center, Semnan University of Medical Sciences, Semnan, Iran \\ ${ }^{2}$ Department of Internal Medicine, Semnan University of Medical Sciences, Semnan, Iran
}

\section{Correspondence to:}

Elaheh Ghods MD, MPH, PhD, Email: ghodsemla@yahoo.com, elaheghods@semums.ac.ir

Received: 15 August 2020 Accepted: 23 November 2020 ePublished: 27 December 2020

Keywords: Tuberculosis, Mycobacterium tuberculosis, Diabetes mellitus

\begin{abstract}
Introduction: Tuberculosis (TB) is one of the most common and lethal infectious diseases.

Objectives: Due to the impact of biological, behavioral, socio-economic factors on the incidence of TB and the risks of the disease, we aimed to investigate the factors affecting TB in TB patients in Semnan.

Patients and Methods: All patients with confirmed TB, who were referred to primary healthcare centers in Semnan for receiving medication, enrolled in the study (2012 to 2016). Data collected from clinical records and in-person interviews. The questionnaire consisted of two parts; the first part was demographic data and the other was related to the risk factors for TB.

Results: The household crowding index was $1.86 \pm 0.88$. Most of patients $(48.1 \%)$ were elderly $(\geq 60$ years old), female $(59.7 \%)$, housewife $(48.1 \%)$, had a pre-diploma education $(63.6 \%)$, low-monthly income $(62.3 \%)$ and married $(63.6 \%)$. Around $13.0 \%$ had diabetes. $20.8 \%$ drug abuser, $16.9 \%$ cigarettes and tobacco smokers, and 1.3\% consumed alcoholic beverages. Besides, $40.3 \%$ were Afghans and $1.3 \%$ had the Pakistani nation.

Conclusion: Identifying and controlling the social, geographical and biological factors affecting the incidence and mortality of TB in different regions can help to formulate appropriate strategies for achieving global goals. These factors differ among native and non-native population.
\end{abstract}

\section{Introduction}

Tuberculosis is a chronic infectious disease with various manifestations. The responsible microorganism, Mycobacterium tuberculosis, is usually transmitted from airborne particles from patients with pulmonary TB to others. After entering the lung alveoli, MT will be entrapped by macrophages and survived there. Just $10 \%$ of infected cases will get the disease. Then the migration of macrophages contaminates the regional lymph nodes. TB has many manifestations, affecting bone, the central nervous system, and many other organ systems, however it is primarily a pulmonary disease (1).

According to the World Health Organization (WHO), the one-third of worldwide population is exposed with $M$. tuberculosis; From about 14 million definite cases of TB disease, $80 \%$ are living in developing countries. Accordingly, in 2014, 9.6 million people infected with $\mathrm{TB}$ and nearly 1.5 million people died from TB (2).

TB, after AIDS, is the second worldwide killer infectious disease, while half of the

\begin{abstract}
Key point
Various causes such as socioeconomic factors and immigration play a significant role in tuberculosis prevalence. By detecting risk factors in tuberculosis (TB) in different geographical areas, we can improve the knowledge for planning and screening of tuberculosis.
\end{abstract}

global burden of death from infectious diseases is associated with three diseases including AIDS, TB and malaria (3).

$\mathrm{TB}$ is at the tenth place in global burden of disease, since it is anticipated that by 2020 , it will maintain its current status in the future as a major source of illnesses (2).

After World War II, there was a great deal of hope for controlling TB by vaccination as a public health tool which later became clear that this tool has a limited protective effect on TB epidemiology. After the availability of TB drugs, the controlling efforts for TB were focused on treatment. It had been anticipated that the appropriate coverage of effective treatment would reduce the incidence of 
$\mathrm{TB}$, however, some recent observations have shown that the impact of the current control strategy (stopping TB) has been less than expected. Today, the emphasis is on the need to expand the TB control strategy and include more preventive efforts. One way to improve the current strategy is to prevent the risk factors of TB (4).

For entering to infection stage in addition to the presence of $M$. tuberculosis, host conditions and environmental factors are also important. Human immunodeficiency virus infection is the strongest risk factor for TB. Other risk factors are including; diabetes, long-term treatment with corticosteroids, immunosuppressive therapy, head and neck cancer, leukemia, lymphoma, malnutrition, silicosis, chronic renal failure and IV drug abuse (1).

The major risk factors for the epidemic TB are poverty, inequality and lack of food security, inappropriate working and living conditions, aging and various chronic diseases (5). In 2015, the WHO (2) introduced the "TB stopping" strategy. One of the main basis of this strategy is social support for reducing poverty and taking effective action for controlling of TB risk factors. The most important factors affecting TB are social determinants of health. Such as, the condition in which a person was born, grows up, lived and worked, as well as disease-fighting systems.

These systems are formed by a group of broader factors, namely, economics, social and politics policy. Namely, lower social classes of community have shown higher prevalence of the disease and accordingly shorter life expectancy. Workplace stress increases the risk of disease developing and people who have more control on their tasks have better health; job security increases well-being and job satisfaction, and unemployment can make symptoms of illness worse. Friendship, good social relationships and strong supportive networks also promote health at home, workplace and society. Patterns of TB disease distribution revealed the structure and wealth of communities (6).

In 2011, the commission of social determinants of health, indicated to inequity and lack of social protection as an effective factor in prevalence of TB (7).

Some of the social determinants of TB are including; occupation, education, biological factors such as age, gender, malnutrition and behavioral factors and habits such as cigarettes and alcohol drinking. In a study on the social determinants data in the United States, there was a relationship between TB and poverty, inequality in income and social capital (8). In another study, a relationship between TB and poverty and education was detected (9). A study in India has shown that diabetes mellitus, alcohol drinking, smoking, and malnutrition also contribute to the TB (10).

Another study pointed the effect of financial crisis on increasing the risk of TB (11). Some of the suggested interventions for reducing the $\mathrm{TB}$ epidemics are: identifying social determinants of $\mathrm{TB}$ and taking measures for reduce the poverty and increasing of food security, improving working and living conditions and taking measures to smoking cessation and alcohol abstinence and prevention from chronic diseases such as diabetes (5).

\section{Objectives}

According to the above, the high global burden of TB and the impact of biological, behavioral, socio-economic factors on its incidence and the risks of the disease, we aimed to determine the factors associated with TB and vulnerable groups so by identifying the risk factors improve the knowledge for planning in this area.

\section{Patients and Methods \\ Protocol}

In a cross-sectional, descriptive study, 77 confirmed cases of TB were selected based on records in Semnan health center. The inclusion criteria were confirmation of TB (diagnosis based on positive culture), 18 years old and more and written informed consent to participate in the study. Incomplete or misleading records were excluded.

\section{Ethical issues}

The research followed the tenets of the Declaration of Helsinki. This study was approved by the research council of Social Determinants of Health Research Center, Semnan University of Medical Sciences (Ethics No. IR.SEMUMS. REC.1395.151). Informed consent was obtained from all participants in the study.

\section{Statistical analysis}

Patients' data were collected from health centers and entered to a checklist. In order to analyze the data, patients were asked to complete the required information. The data were inserted to datasheet and analyzed in statistical software SPSS version 23. Numerical and categorical variables were reported as mean \pm standard deviation and number (percentage), respectively.

\section{Results}

In this study, all 77 patients with confirmed TB (by culture) who were registered in health centers in Semnan during the years 2011 to 2016 enrolled in the study. The annual incidence of disease in the first year was 5.8/100000 and in the last year $8.1 / 100000$ and 5 years incidence rate was 9.1/100000.

The mean age was $87.86 \pm 56.88$ years (minimum 18 and maximum 98 years old). 37 patients (48.1\%) had 60 years old or more. 46 patients $(59.7 \%)$ were female and $31(40.3 \%)$ male. 10 patients $(13.0 \%)$ had diabetes and 67 patients $(87.0 \%)$ had not other disease. There was no case of HIV infection. Two patients (2.6\%) had a history of taking immunosuppressive drugs and three patients (3.9\%) had a history of chemotherapy. One person (1.3\%) was immunocompromised (primary immunodeficiencies).

Sixteen patients $(20.8 \%)$ were drug abusers and 60 (77.9\%) had no history of drug abuse. One patient (1.3\%) also had a history of drug abuse that had abandoned. 
Thirteen patients (16.9\%) were smoker. Five patients (6.5\%) also had a history of smoking and tobacco that had abandoned. One patient (1.3\%) was drinking alcohol and one person (1.3\%) had a history of alcohol abuse.

Around 30 patients were housewives, 20 workers and 10 retired, and the rest were self-employment and employees or students. The educational level in 49 patients was below of diploma. About 45 patients had Iranian nationality and $31(40.3 \%)$ were Afghan and one patient (1.3\%) had Pakistani nationality.

The mean $( \pm \mathrm{SD})$ of patients income was $9162337 \pm 3091203$ Rls. (maximum; 17000000 , minimum; $3000000 \mathrm{Rls}$ ). Most people with low income $(62.3 \%)$. Four patients $(2.5 \%)$ were under the coverage of relief foundation and two patients $(2.6 \%)$ under the coverage of welfare organization. Of the patients, 64 (83.1\%) were residents of the city and 13 (rural) residents.

The mean $( \pm \mathrm{SD})$ of the family members of the patients was $3.17 \pm 1.59$ (minimum; 1 , maximum; 9). The mean $( \pm \mathrm{SD})$ of number rooms in patients' houses was $1.81 \pm 0.59$ (minimum; 1 room and maximum; 4 rooms). The ratio of person to room was 1.86 (minimum rate was 0.50 and maximum; 5.00).

The mean $( \pm \mathrm{SD})$ of the patients' body mass index (BMI) was $23.15 \pm 4 \mathrm{~kg} / \mathrm{m}^{2}$, ranging from $16.89-34.16 \mathrm{~kg} / \mathrm{m}^{2}$. The BMI in most patients $(55.8 \%)$ was in normal range (18.5 to $24.9 \mathrm{~kg} / \mathrm{m}^{2}$ ).

\section{Discussion}

Environmental factors and some of the underlying diseases contribute to the increased risk of $\mathrm{TB}$, and the identification of effective factors in each area will help in disease control and prevention.

The number of patients with TB in Semnan was increased between 2010 and 2015. The incidence of TB in different parts of Iran has not shown a significant decrease in the last two decades (12). Prospective research in our country has even predicted a steeper slope for TB prevalence from 2009 to 2014 (13). The achievement of the WHO's goals in reducing the incidence and mortality of TB indicated to carefully assess the factors affecting the incidence and mortality of TB (2).

Studies in developing countries show the need for more attention to epidemiological issues and social components along with clinical measures to achieve WHO goals in anti-TB affairs $(7,14)$.

Different regions of Iran are various in terms of health and infection indicators. Such research can therefore help achieve the goals of regional programs for health improvement. The geographical locations of Iran and its neighborhood with Afghanistan, Pakistan, Iraq and the newly independent northern states including Tajikistan, Armenia, Turkmenistan and Azerbaijan and also regional unrest in recent years indicate a necessity to determine the factors influencing the incidence, prevalence, pattern of disease, disability and mortality of $\mathrm{TB}$ and reviewing of current programs with regard to the variety of factors and their impact in different areas.

In this study, more than $40 \%$ of patients with $\mathrm{TB}$ in Semnan are non-native (immigrant from Afghanistan). The regression model obtained in 2016 identified migration as one of the main causes of TB (15). The WHO report in 2017 indicated that the incidence of TB in Afghanistan was 159 per 100000 (2). The highest incidence of TB in Iran is in Sistan and Baluchistan province due to neighborhood with Afghanistan and Pakistan $(12,16)$.

A systematic review of the social determinants of health between 1990-2017 reveals differences in these indices between native and non-native population (17). Investigating the causes and factors affecting the high prevalence of $\mathrm{TB}$ in Golestan province indicates the determining role of the origin of this region (Turkmen) (16). In the present study, a significant number of TB patients were not native. However, lower prevalence of the disease in central areas, including Semnan, does not allow for analysis of its trend.

The second wave of TB prevalence has a strong association with emerging diseases such as AIDS and immunodeficiency and has been identified as the leading cause of mortality in AIDS. Worldwide incidence of TBAIDS was about $12 \%$ and about half of cases originated from Asian-Pacific region (18). Our study reported only one person with AIDS (1.3\%). Comparing incidence of TB in HIV cases in different countries also indicated lower percentage of this co-infection in Iran. Therefore, AIDS could not be a major determinant of TB burden in our country (19).

The mean age of patients with TB in our study was 56.78 years which had substantial difference compared to other studies. The mean age of patients with TB in Khazaei study (20) in Hamadan was the same as our study, 50-51 yeares in north of Iran highlighted by Babamahmoodi et al (21) and 42 years in Ardabil province (22), which were less than in compare of mean age of patients in our study.

In our study, $48.1 \%$ of patients were elderly (60 years age and older); the highest incidence of TB in the study by Khazaee et al in Hamadan province during 2005 to 2011 was over 60 years (20). So, TB incidence has shown strong age association (23). Even mean age TB cases of Sunnetcioglu et al in Turkey (24) and Atif et al in Pakistan (25) were less than 40 years.

It is a well-known fact that the immune system is suppressed by aging and the chance of involvement of TB infection increases and it can be considered as a potential risk factor for TB. Odds of delay in diagnosis, drug interaction and resistance, frailty and its complication, poverty, recurrence of TB be increased by aging (26-28). Another point is that older people are less aware of health warnings and therefore have less attention to health issues; besides, older people are more exposed to predisposing risk factors such as various chronic diseases, including 
diabetes, which, by weakening the immune system, makes it easier involvement of TB.

The impact of urbanization on TB has been investigated in several studies. Population density increases the chances of being exposed to air pollution, more environmental pollutants and pathogens (29-31). Abundant immigration increases vulnerable populations on the outskirts of cities, which have low level of health and social services coverage and as a floating population should be given greater attention in infectious diseases such as TB (30). In this study, 64 out of 77 patients (83\%) were from urban areas, more than similar studies in Salmas 2006-2015 (40\%) (32), Hamadan (60\%) (20), North of Iran (53.8\%) (21). Of course, changes in recent years in the national divisions in Semnan province (increasing cities and decreasing rural areas) can also play a role in this difference. On the other hand, TB screening and treatment system - DOTS (Directly Observed Treatment Short) course - in urban areas seems to be more effective.

Therefore, it is estimated that urban residents, probably due to more follow-up and access to more diagnostic methods, the chance of diagnosis is better and of course, it is expected that the prevalence of TB was higher in urban than rural areas.

Gender-related differences in immune response to Mycobacterium tuberculosis (33), location of involvement, diagnosis, access to health care, incomplete follow-up and treatment have been studied in various studies (34-37). In low-income countries such as Brazil, men are more likely to be diagnosed, treated incompletely, and died from pulmonary TB (36). In contrast, research findings in Bangladesh have shown a significantly longer delay in diagnosis and entry into the DOTS program in women than in men (34). Extrapulmonary TB and negative smear have also been reported significantly higher in women than men (38). While epidemiologic study in Iran showed no significant gender differences in extrapulmonary TB (22).

In our study, approximately $60 \%(\mathrm{n}=46)$ of the patients were female. Sex differences in TB prevalence were controversial in different regions, ratio of female patients was $38 \%$ in north of Iran, which was similar to our study (37). However, in Nepal, female gender was identified as a major risk factor for TB (34). In a study in Hamedan province during the years 2005 to 2011 , the results showed that the incidence of TB in women was more than that in men (21).

Khazaei et al, in a population-based study found that non-pulmonary $\mathrm{TB}$ was significantly higher in women than men (20). This finding is similar to our study. This issue can be due to the job and also the status of the woman in the different culture and nations, since most women with TB in the present study were housewives, and given the nature of the TB which is a recurrent and communicable disease, probably due to lower awareness of housewives about the prevention and transmission of the disease, they have not been protecting themselves against the risk factors associated with TB. However, we did not aim to study the knowledge and attitude of people about TB; Therefore other studies can be conducted with this purpose. Recognizing the risk factors for the onset or recurrence of TB and, consequently, educating about avoiding these risk factors is necessary.

This issue can be due to the job and also the status of the woman in the different culture and nations, since most women with $\mathrm{TB}$ in the present study were housewives, and given the nature of the TB which is a recurrent and communicable disease, probably due to lower awareness of housewives about the prevention and transmission of the disease, they have not been protecting themselves against the risk factors associated with TB; however, we do not aimed to study the knowledge and attitude of people about TB, but other studies can be conducted with this purpose. Recognizing the risk factors for the onset or recurrence of TB and, consequently, educating about avoiding from these risk factors is necessary.

In our study, 10 patients (13.0\%) were diabetic. Sixteen patients $(20.8 \%)$ were drug abusers, 13 patients $(16.9 \%)$ had a history of smoking and tobacco, and one person (1.3\%) was drinking alcohol. One (1.3\%) had a history of drug abuse who quitted. Five people (6.5\%) also had a history of smoking and tobacco that quitted. One person (1.3\%) had a history of drug abuse who quitted.

In a descriptive study conducted in Mazandaran province, Abedi et al found that diabetes and smoking can be considered as an important risk factor for TB. Therefore, in the case of respiratory symptoms, TB should be considered as one of the important differential diagnoses (37).

In addition, Sarvi et al studied the epidemiological data of TB in 28 provinces of Iran from 2001-2006. Findings showed that reducing the amount of smoking had a positive impact on the reduction of TB in Iran (15).

Gupta and colleagues found that diabetes mellitus, smoking and alcohol are the most important risk factors for TB; it has been recommended that patients with diabetes mellitus who have pulmonary symptoms should be screened for TB (10).

Diabetes is associated with increase risk of active TB, and diagnosis ad control of diabetes could have a major impact on the control of TB $(37,38)$.

In this study, $9.1 \%$ of patients had malnutrition (BMI $<18.5 \mathrm{~kg} / \mathrm{m}^{2}$ ). In a large epidemiological study in more than 200 countries, the malnutrition rate in the Iranian population was $12.1 \%$ (39).

Other studies found that there is a two-way link between malnutrition and TB; malnutrition is a health outcome and thus risk factor for diseases; on the other hand, malnutrition and its immunosuppressive effects can result in complications and drug-resistant TB. Therefore, 
screening for the prevention and treatment of malnutrition can play an important role in reducing the burden of TB in the community $(10,15,17)$.

Most of patients in our study were low income (62.3\%). Six patients $(7.8 \%)$ were supported by supportive organizations. In another study, the results of the function estimation showed that increased per capita income, health care, education and sport expenditure, have a positive impact on the reduction of TB in Iran (15). Low-income, possibly due to the inability of the people to taking of appropriate health care, and the disruption of social and health conditions, including malnutrition, can lead the person to a defective cycle and increase the risk of TB. Other researchers also found malnutrition and poverty is a major risk factor for the spread of TB $(1,4,7)$.

Several studies highlighted strong correlation between TB and poverty; Therefore poverty could increase the incidence of $\mathrm{TB}$, and the incidence of $\mathrm{TB}$ is associated with rising poverty. Additionally, a significant fraction of the annual income of the family spends on TB treatment $(12,14,16)$. This matter confirms the importance of economic poverty effect on TB, which is similar to our study results. Besides, economic poverty leads to the lack of collaboration of the patient with TB during the treatment period, and thus increases the possibility of transmitting the disease to others, especially family members and relatives, and consequently, increases the prevalence of TB.

Jiba et al indicated that social determinants affecting the discontinuation of treatment for TB are categorized into 6 main themes: lake of social support, notoriety duo to TB, work place issues, poverty, imprisonment and drug abuse during the treatment period (40). These points emphasize the importance of the role of poverty in TB. Arinaminpathy et al have shown a strong association between improving the economic status and the occurrence of TB, therefore, a relationship between the economic productivity losses during the recession and the increase in incidence of TB was seen (11).

Our study results showed that the majority of patients had high school degree and lower (63.6\%). Epidemiological studies evaluated the social determinants of TB and found that illiteracy, unemployment and low welfare increases the risk of TB $(14,15,17)$. These findings are similar to our study. A recent study has shown that TB is associated with low education, poverty and high population density (9). Low-awareness about the methods of disease transmission as well as its protective methods can explain the high prevalence of TB in people with low educational levels.

In this study, there were some limitations. One of the limitations of our study was the lack of control group to evaluate the chance ratio of related risk factors in the incidence of TB and, consequently, to improve the outcome. One of the other limitations was the inability of the patients in recalling a number of variables. On the other hand, there are several variables that affect TB, which, of course, we could not evaluate all of them in our study.

\section{Conclusion}

Overall, the results of this study showed that various causes such as biological, behavioral, socioeconomic and economic factors affect TB prevalence, and immigration also plays a significant role in TB. The most common risk factors for occurrence of TB in our study were aging, diabetes and drug abuse, which are different from those in the world such as AIDS and poverty, and therefore in this geographical area special attention is needed in these groups for screening of TB.

\section{Suggestions for future studies}

This study was conducted in a limited population and more studies are recommended, especially in Iranian societies, by considering limitations.

TB screening is recommended for early patients identification at high risk population, in order to manage early treatment and control and more satisfactorily.

\section{Limitations of the study}

One of the most important limitations in this study was that most of the patients were from low socio-economic classes, and this was an obstacle to following up, which was resolved by several follow-ups.

Authors' contribution

NSK, MNZ, and EG prepared grant submissions for the project and participated in the planning, preparation and approval of the original questionnaire. ES conducted the questionnaire survey and data collection. All authors contributed to the writing of the article, and read and approved the final manuscript

Conflicts of interest

The authors declare no conflicts of interest.

Ethical considerations

Ethical issues (including plagiarism, data fabrication, double publication) have been completely observed by the authors.

Funding/Support

This study was supported financially by Semnan University of Medical Sciences (Grant \#A-10-289-5).

\section{References}

1. Raviglione MC. Tuberculosis. In: Longo DL, Fauci AS, Kasper DL, Hauser SL, Jameson JL, Loscalzo J, editors. Harrison's Principles of Internal Medicine. 20th ed. New York, N.Y: McGraw-Hill Medical; 2015. p. 1236-58.

2. WHO. World Health Organization. Tuberculosis 2018.Available from: https://www.who.int/news-room/fact-sheets/detail/ tuberculosis.

3. Wallace RB. Maxcy-Rosenau-Last Public Health and Preventive Medicine. 15th ed. New York, NY: McGraw-Hill; 2008.

4. Lonnroth K, Jaramillo E, Williams BG, Dye C, Raviglione M. Drivers of tuberculosis epidemics: the role of risk factors and social determinants. Soc Sci Med. 2009;68:2240-6. doi: 10.1016/j. socscimed.2009.03.041.

5. Uplekar M, Weil D, Lonnroth K, Jaramillo E, Lienhardt C, Dias HM, 
et al. WHO's new End TB Strategy. Lancet. 2015;385:1799-801. doi: 10.016/S0140-6736(15)60570-0.

6. Wilkinson RG, Marmot M. Social determinants of health: the solid facts. World Health Organization; 2003.

7. Hargreaves JR, Boccia D, Evans CA, Adato M, Petticrew M, Porter $\mathrm{JDH}$. The social determinants of tuberculosis: from evidence to action. Am J Public Health. 2011;101:654-62. doi: 10.2105/ AJPH.010.199505.

8. Holtgrave DR, Crosby RA. Social determinants of tuberculosis case rates in the United States. Am J Prev Med. 2004;26(2):159-62. doi: 10.1016/j.amepre.2003.10.014.

9. Cantwell MF, McKenna MT, McCray E, Onorato IM. Tuberculosis and race/ethnicity in the United States: impact of socioeconomic status. Am J Respir Crit Care Med. 1998;157:1016-20. doi: 10.164/ ajrccm.157.4.9704036

10. Gupta S, Shenoy VP, Mukhopadhyay C, Bairy I, Muralidharan S. Role of risk factors and socio-economic status in pulmonary tuberculosis: a search for the root cause in patients in a tertiary care hospital, South India. Trop Med Int Health. 2011;16:74-8. doi: 10.1111/j.365-3156.2010.02676.x.

11. Arinaminpathy $\mathrm{N}$, Dye $\mathrm{C}$. Health in financial crises: economic recession and tuberculosis in Central and Eastern Europe. J R Soc Interface. 2010;7:1559-69. doi: 10.098/rsif.2010.0072.

12. Metanat M, Sharifi-Mood B, Alavi-Naini R, Aminianfar M. The epidemiology of tuberculosis in recent years: Reviewing the status in south-eastern Iran. Zahedan J Res Med Sci. 2012;13:e93704.

13. Moosazadeh M, Nasehi M, Bahrampour A, Khanjani N, Sharafi S, Ahmadi S. Forecasting tuberculosis incidence in iran using boxjenkins models. Iran Red Crescent Med J 2014;16:e11779-e. doi: 10.5812/ircmj.11779.

14. MacNeil A, Glaziou P, Sismanidis C, Maloney S, Floyd K. Global epidemiology of tuberculosis and progress toward achieving global targets-2017. MMWR Morb Mortal Wkly Rep. 2019;68:263.

15. Sarvi F, Momenian S, Khodadost M, Pahlavanzadeh B, Nasehi M, Sekhavati E. The examination of relationship between socioeconomic factors and number of tuberculosis using quantile regression model for count data in Iran 2010-2011. Med J Islam Repub Iran. 2016;30:399.

16. Rafiee S, Besharat S, Jabbari A, Golalipour F, Nasermoaadeli A. Epidemiology of tuberculosis in northeast of Iran: a populationbased study. Iran J Med Sci. 2009;34(3):193-7.

17. Cormier M, Schwartzman K, N'Diaye DS, Boone CE, Dos Santos AM, Gaspar J, et al. Proximate determinants of tuberculosis in Indigenous peoples worldwide: a systematic review. Lancet Glob Health. 2019;7:e68-e80. doi: 10.1016/S2214-109X(18)30435-2.

18. Trinh QM, Nguyen HL, Nguyen VN, Nguyen TV, Sintchenko V, Marais BJ. Tuberculosis and HIV co-infection-focus on the AsiaPacific region. Int J Infect Dis. 2015;32:170-8.

19. Pourakbari B, Mamishi S, Banar M, Keshtkar AA, Mahmoudi S. Prevalence of TB/HIV co-infection in Iran: a systematic review and meta-analysis. Ann Ig. 2019;31:333-48. doi: 10.7416/ ai.2019.2295.

20. Khazaei S, Roshanaei G, Saatchi M, Rezaeian S, Zahiri A, Bathaei SJ. The epidemiological aspects of tuberculosis in Hamadan province during 2005-11. Int J Health Policy Manag. 2014;2:7580. doi: 10.15171/ijhpm.2014.18.

21. Babamahmoodi F, Alikhani A, Yazdani Charati J, Ghovvati A, Ahangarkani F, Delavarian L, et al. Clinical epidemiology and paraclinical findings in tuberculosis patients in north of Iran. Biomed Res Int. 2015;2015:381572. doi: 10.1155/2015/381572.

22. Pirzadeh A, Habibzadeh S, Malekmohammadi R. Changing trend of tuberculosis indicators in Ardabil province, 2005-2012: a province in north-west of Iran. IJMDC. 2020;4:572-575. doi:10.24911/IJMDC.51-1558272153

23. Marvi A, Asadi-Aliabadi M, Darabi M, Rostami-Maskopaee F,
Siamian H, Abedi G. Silent changes of tuberculosis in Iran (20052015): A joinpoint regression analysis. J Family Med Prim Care. 2017;6:760-765. doi: 10.4103/jfmpc.jfmpc_190_17.

24. Sunnetcioglu A, Sunnetcioglu M, Binici I, Baran AI, Karahocagil MK, Saydan MR. Comparative analysis of pulmonary and extrapulmonary tuberculosis of 411 cases. Ann Clin Microbiol Antimicrob. 2015;14:34. doi: 10.1186/s12941-015-0092-2.

25. Atif M, Anwar Z, Fatima RK, Malik I, Asghar S, Scahill S. Analysis of tuberculosis treatment outcomes among pulmonary tuberculosis patients in Bahawalpur, Pakistan. BMC Res Notes. 2018;11:370. doi: 10.1186/s13104-018-3473-8.

26. Moosazadeh M, Bahrampour A, Nasehi M, Khanjani N. The incidence of recurrence of tuberculosis and its related factors in smear-positive pulmonary tuberculosis patients in Iran: A retrospective cohort study. Lung India. 2015;32:557-60. doi: 10.4103/0970-2113.168113.

27. Negin J, Abimbola S, Marais BJ. Tuberculosis among older adultstime to take notice. Int J Infect Dis. 2015;32:135-7. doi: 10.1016/j. ijid.2014.11.018

28. Li H, Javid B. Antibodies and tuberculosis: finally coming of age? Nat Rev Immunol. 2018;18:591-596. doi: 10.1038/s41577-0180028-0.

29. Kelmelis KS, Pedersen DD. Impact of urbanization on tuberculosis and leprosy prevalence in medieval Denmark. Anthropol Anz. 2019;76:149-166. doi: 10.1127/anthranz/2019/0962.

30. Li X, Yang Q, Feng B, Xin H, Zhang M, Deng Q, et al. Tuberculosis infection in rural labor migrants in Shenzhen, China: Emerging challenge to tuberculosis control during urbanization. Sci Rep. 2017;7:4457. doi: 10.1038/s41598-017-04788-1.

31. Mokhtar KS, Rahman NHA. Urbanisation process and the prevalence of tuberculosis in Malaysia. GEOGRAFIA OnlineMalaysian Journal of Society and Space. 2017;11:123-7.

32. Taghinejad J, Sadeghi Oroumiyeh A, Barati B, Karimi S, Jahantab M, Molayi Kohneshahri S. Epidemiologic study of tuberculosis during 2006-2015 in Salmas, Iran. Int J Health Life Sci. 2018;4(2):e80210.

33. Sathyamoorthy T, Sandhu G, Tezera LB, Thomas R, Singhania A, Woelk $\mathrm{CH}$, et al. Gender-dependent differences in plasma matrix metalloproteinase-8 elevated in pulmonary tuberculosis. PloS One. 2015;10:e0117605. doi: 10.1371/journal.pone.

34. Horton KC, MacPherson P, Houben RM, White RG, Corbett EL. Sex differences in tuberculosis burden and notifications in lowand middle-income countries: a systematic review and metaanalysis. PLoS Med. 2016;13:e1002119. doi: 10.1371/ journal. pmed.1002119.

35. Yates TA, Atkinson SH. Ironing out sex differences in tuberculosis prevalence. Int J Tuberc Lung Dis. 2017;21:483-4. doi: 10.5588/ ijtld.17.0194.

36. Hertz D, Schneider B. Sex differences in tuberculosis. Semin Immunopathol. 2019;41:225-237.

37. Abedi S, Moosazadeh M, Tabrizi R, Afshari M, Nezammahalleh A, Akbari M. The impact of diabetics and smoking on gender differences of smear positive pulmonary tuberculosis incidence. Indian Journal of Tuberculosis. 2019;66(3):353-7.

38. Silva DR, Muñoz-Torrico M, Duarte R, Galvão T, Bonini EH, Arbex $\mathrm{FF}$, et al. Risk factors for tuberculosis: diabetes, smoking, alcohol use, and the use of other drugs. Jornal Brasileiro de Pneumologia. 2018 Apr;44(2):145-52.

39. NCD Risk Factor Collaboration. Trends in adult body-mass index in 200 countries from 1975 to 2014: a pooled analysis of 1698 population-based measurement studies with $19 \cdot 2$ million participants. Lancet. 2016;387:1377-96.

40. Jiba M, Zamani-Alavije F, Araban M, Salahshoori A, Jiba M, Haroni J. Social factors influencing treatment interruption in Tuberculosis Patients: A qualitative study. Journal of Health in the Field. 2015;2:26-34. 